\title{
La banda gástrica contribuye a la remisión clínica de la diabetes tipo 2 en individuos obesos
}

\author{
Laparoscopic banding surgery contributes to clinical remission of type 2 diabetes in obese individuals
}

Dixon J y col. JAMA, January 23, 2008; 299:316-323.

\section{Objetivo}

Determinar si la pérdida de peso obtenida a través de la cirugía de "banding" contribuye a un mejor control de los pacientes con diabetes tipo 2.

\section{Diseño, lugar y pacientes}

Ensayo clínico abierto controlado y aleatorizado, llevado a cabo en el Centro Universitario de Investigación en Obesidad de Australia entre 2002 y 2004. Incluyó 60 pacientes de 20 a 60 años de edad (reclutados mediante una aviso de un diario) con un índice de masa corporal (IMC) entre 30 y 40, con diagnostico documentado de diabetes tipo 2 durante los dos años previos, sin evidencia de retinopatía ni nefropatía y capaces de entender y cumplir el estudio. Fueron aleatorizados en dos grupos: 1) a los 30 del grupo control se los asignó a un dispositivo interdisciplinario de tratamiento clínico (TnoQ); 2) a los otros 30, sumado a dicho dispositivo, se les indicó cirugía (TQ).

\section{Intervención y medición de resultados principales}

Para el grupo control, el dispositivo interdisciplinario de tratamiento clínico implicaba libre acceso para consultar a cualquier miembro del equipo tratante (médicos generalistas, nutricionistas, enfermeros, etc.). Los pacientes debían realizar un mínimo de una consulta cada seis meses. Se les ofreció tratamiento individualizado con hipoglucemiantes, un programa de actividad física (10.000 pasos por día más 200 minutos semanales de actividad física aeróbica de intensidad moderada y ejercicios de resistencia) y una dieta. El paciente podía optar por una dieta de muy bajas calorías y tomar medicación para bajar de peso (ej. sibutramina). Al grupo asignado a cirugía, además del dispositivo clínico interdisciplinario, se les ofreció una cirugía de "banding" por vía laparoscópica al mes del la aleatorización. Fueron evaluados cada cuatro a seis semanas, con un eventual ajuste de la banda según criterio clínico. El análisis fue por intención de tratar y el resultado principal evaluado, la proporción de pacientes que logró remisión de la diabetes tipo 2 (glucemia en ayunas menor a $126 \mathrm{mg} / \mathrm{dL}$ y hemoglobina glicosilada menor a 6,2\%) sin necesidad de hipoglucemiantes orales.

\section{Resultados principales}

Ver tabla 1.

Tabla 1: remisión clínica de la diabetes tipo 2 a los dos años de seguimiento de acuerdo a la estrategia ofrecida (tratamiento no quirúrgico con o sin el agregado de cirugía de "banding").

\begin{tabular}{l|c|c|c|c|c}
\multirow{2}{*}{ Resultados } & \multicolumn{2}{|c|}{$\begin{array}{c}\text { Ambos grupos recibieron } \\
\text { tratamiento no quirúrgico }\end{array}$} & \multirow{2}{*}{ RR (IC95\%) } & RRA & NNT \\
\cline { 2 - 3 } & $\begin{array}{c}\text { Control } \\
(n=31)\end{array}$ & $\begin{array}{c}\text { Cirugía } \\
(n=30)\end{array}$ & & & \\
\hline Remisión de la diabetes & $4(13 \%)$ & $22(73 \%)$ & $5,5(2,2$ a 14$)$ & $60 \%$ & 1,7 \\
\hline Seguimiento & $87 \%$ & $97 \%$ & & & \\
\hline
\end{tabular}

RRA: reducción del riesgo absoluto. NNT: número de pacientes que es necesario someter a cirugía de Banding para lograr una remisión adicional de diabetes tipo 2 .

\section{Conclusiones}

A los dos años de seguimiento, el agregado de una cirugía de "banding" al tratamiento no quirúrgico se asoció a una mayor remisión de la diabetes tipo 2 .

Palabras claves: remisión, diabetes tipo 2, cirugía de "banding" gástrico. Key words: remission, type 2 diabetes, gastric banding surgery.

Fuente de financiamiento: National Health and Medical Research Council, Allergant Health, Novartis Australia.

\section{Comentario}

La obesidad y la diabetes tipo 2 son dos problemas de salud fuertemente relacionados que generan deterioro de la calidad de vida de quienes los padecen e importantes gastos en salud. Si bien el control del peso contribuye a un mejor manejo de la glucemia y reduce la morbi-mortalidad; actualmente los tratamientos médicos son poco efectivos, perfilándose prometedora la cirugía laparoscópica de "banding".

De acuerdo a información previa con la que contamos ${ }^{1,2}$, el estudio que hemos resumido es el primer ensayo clínico con un seguimiento de dos años que documentó individualmente en diabéticos tipo 2 obesos, la eficacia del agregado de un procedimiento quirúrgico al manejo clínico habitual (mayor remisión de la diabetes, mejores niveles de colesterol HDL, menor resistencia a la insulina y menor necesidad de hipoglucemiantes, hipolipemiantes y antihipertensivos). Sin embargo, tiene la limitación de haber seleccionado una población con reciente diagnóstico, haber incluido sólo 60 pacientes y, por el momento, sólo puede brindar resultados hasta dos años de seguimiento.

\section{Conclusiones de la comentadora}

A pesar de que estos resultados son impactantes, todavía desconocemos la efectividad en el largo plazo de este tipo de estrategia, por lo que esperamos con ansias la aparición de nuevas publicaciones que aporten mayor evidencia en esta área de tanto impacto en la salud pública.

Gabriela Buela [ Servicio de Medicina Familiar y Comunitaria. Hospital Italiano de Buenos Aires. gabriela.buela@ hospitalitaliano.org.ar ]

Buela G. La banda gástrica contribuye a la remisión clínica de la diabetes tipo 2 en individuos obesos. Evid. Actual. Práct. Ambul; 12(1): 10, Ene-Mar 2009. Comentado de: Dixon $\mathbf{J}$ y col. Adjustable gastric banding and conventional therapy for DBT II: A randomized controlled trial. JAMA. 2008 Jan 23;299(3):316-23. PMID: 18212316. Disponible en URL: http://jama.ama-assn.org/cgi/content/full/299/3/316 (último acceso 31/01/09).

\section{Referencia}

1. Cuneen S. Review of meta-analytic comparisons of bariatric surgery with a focus on laparoscopic adjustable gastric banding. Surg Obes Relat Dis. 2008 May-Jun; $4(3$ Suppl):S47-55.

2. O'Brien. Treatment of mild to moderate obesity with laparoscopic adjustable gastric banding or an intensive medical program: a randomized trial. Ann Intern Med. 2006 May 2; 144(9):625-33. 